\title{
Traquelectomía radical laparoscópica en pacientes con cáncer de cuello uterino en estado IBI en el Instituto de Cancerología - Las Américas, Medellín, Colombia
}

\author{
Lina Echeverri ${ }^{1}$, Claudia López ${ }^{2}$, Gabriel Jaime Rendón ${ }^{1}$, Gonzalo Ángel ${ }^{1}$, René Pareja. ${ }^{1}$ \\ ${ }^{1}$ Ginecólogo Oncólogo, Departamento de Ginecología Oncológica, Instituto de Cancerología - Las Américas. ${ }^{2}$ Gineco- \\ Obstetra, Departamento de Ginecología Laparoscópica, Clínica Las Américas. Medellín, Colombia.
}

\section{RESUMEN}

Objetivo: Revisar nuestra experiencia en traquelectomía radical laparoscópica en cáncer de cérvix en estado temprano. Métodos: Estudio observacional descriptivo retrospectivo de las pacientes que se sometieron a traquelectomía radical laparoscópica en el Instituto de Cancerología - Las Américas, Medellín, Colombia, entre Mayo de 2009 y Enero 2015. Resultados: Se completó satisfactoriamente el procedimiento en 13 pacientes en estadío IB1, $12(92 \%)$ tuvieron tumores menores a $2 \mathrm{~cm}$ y una recibió quimioterapia neoadyuvante por un tumor de $3 \mathrm{~cm}$. El tipo histológico más frecuente fue el escamoso en 7 pacientes (54\%). En 5 pacientes (38\%) no se evidenció enfermedad residual luego del procedimiento. El tiempo quirúrgico promedio fue de $240 \mathrm{~min}$ (130-340 min); la mediana de pérdida sanguínea fueron $100 \mathrm{ml}$ (50-200 $\mathrm{ml}$ ). Dos pacientes tuvieron cistotomía incidental durante la cirugía, reparadas por vía laparoscópica, una de ellas desarrollo una fístula vésico-vaginal que cerró espontáneamente. Ninguna paciente requirió transfusión sanguínea ni conversión a laparotomía. La estancia hospitalaria promedio fue de 1 día (1-3). Dos pacientes requirieron histerectomía laparoscópica. No se han reportado recurrencias ni embarazos con un seguimiento promedio 27,9 meses (8,3-64,4). Conclusión: La traquelectomía radical laparoscópica es factible y se puede realizar de manera segura en un país en desarrollo, en pacientes bien seleccionados, con cáncer de cérvix temprano que desean preservar su fertilidad. Los tiempos de seguimiento aún son cortos para conclusiones definitivas sobre el resultado obstétrico. La seguridad oncológica parece similar a la de la traquelectomía abdominal y/o vaginal.

\section{PALABRAS CLAVE: Neoplasias del cuello uterino, preservación de la fertilidad, traquelectomía radical laparoscópica}

\section{SUMMARY}

Objective: To review our experience with laparoscopic radical trachelectomy in patients with early-stage cervical cancer. Methods: We performed a observational descriptive retrospective review of all patients who underwent a laparoscopic radical trachelectomy at the Instituto de Cancerología - Las Americas, Medellin, Colombia, between May 2009 and January 2015. Results: 13 patients completed surgery, 12 patients (92\%) were diagnosed as IB1 with tumor size less than $2 \mathrm{~cm}$, and one patient received neoadjuvant chemotherapy due to a $3 \mathrm{~cm}$ cervical tumor; histology was squamous cell carcinoma in 7 patients (54\%). Five patients (38\%) showed no residual disease. The mean surgical time was $240 \mathrm{~min}$ (130-340 $\mathrm{min})$; the estimated blood loss was $100 \mathrm{ml}(50-200 \mathrm{ml})$. Two patients had a cystotomy during surgery; one of them developed a vesicovaginal fistula that healed spontaneously. No transfusions were given. No conversions were made. Mean hospital stay 1 day (1-3 day). 2 patients required laparoscopic hysterectomy. To date, no recurrence 
or pregnancies has been recorded with the median follow up 27.9 months (8.3-64.4 month). Conclusion: Laparoscopic radical trachelectomy is feasible and can be performed safely in a developing country in wellselected patients with early cervical cancer who wish to preserve their fertility. Follow up times are still too short to drive definitive conclusions on obstetric results. The oncological safety appears similar to that of the abdominal trachelectomy and/or vaginal.

\section{KEY WORDS: Uterine cervical neoplasm, fertility preservation, laparoscopic radical trachelectomy}

\section{INTRODUCCIÓN}

El tratamiento quirúrgico habitual para el cáncer de cuello uterino estado IB1 es la histerectomía radical más la linfadenectomía pélvica, con la consecuente pérdida de la capacidad para concebir. En 1987, Daniel Dargent, propuso la traquelectomía radical vaginal con linfadenectomía pélvica laparoscópica para pacientes con tumores confinados al cérvix; su trabajo fue publicado en 1994 y se convirtió en un hito en cuanto a preservación de fertilidad (1). A la fecha existen más de 1000 casos publicados en la literatura, con un resultado oncológico similar a la histerectomía radical con recurrencias y mortalidad de $4 \%$ y $2 \%$ respectivamente, y tasa de embarazos cercana al $44 \%$ (2). Debido a que no todos los ginecólogos oncólogos tienen entrenamiento en cirugía radical vaginal o en laparoscopia, en 1997, Smith introduce la traquelectomía radical abdominal que es una técnica más sencilla y más parecida a la histerectomía radical abdominal (3). Recientemente nuestro grupo, publicó una revisión sistemática de la literatura al respecto contabilizando casi 500 casos con excelente resultado oncológico, pero con resultados obstétricos inferiores a los de su contraparte vaginal (4).

Con el auge de la cirugía mínimamente invasiva en ginecología oncológica, en 2003, Lee y cols reportan la primera traquelectomía radical laparoscópica (5) y Persson en 2008 describe la primera traquelectomía radical robótica (6).

Actualmente, las guías de manejo de tumores ginecológicos de la National Comprehensive Cancer Network (NCCN), incluyen a la traquelectomía radical como alternativa en mujeres con cáncer de cuello uterino en estado temprano, que desean preservar su fertilidad (7).

Nuestro propósito es describir nuestra serie de casos y hacer una revisión de los artículos reportados en la literatura.

\section{PACIENTES Y MÉTODOS}

Luego de obtener la aprobación por parte del comité de ética médica e investigación del Instituto de Cancerología - Las Américas, se realizó de manera retrospectiva una revisión de las historias clínicas de las pacientes sometidas a traquelectomía radical laparoscópica entre Mayo de 2009 y Enero 2015. Se usaron como criterios de inclusión los siguientes: diagnóstico confirmado por biopsia o conización de carcinoma invasor de cuello uterino, histología escamocelular, adenocarcinoma o adenoescamoso, estado clínico IB1 según la International Federation of Gynecology and Obstetrics (FIGO), ausencia de compromiso ganglionar pélvico o para-aórtico en las imágenes disponibles, ausencia de enfermedad a distancia, fuerte deseo de preservar la fertilidad y que el procedimiento fuera realizado por laparoscopia.

Las variables a considerar fueron: edad, índice de masa corporal, estado clínico, tamaño tumoral, histología, compromiso linfovascular, tiempo quirúrgico, sangrado intraoperatorio, necesidad de transfusión, complicaciones intraoperatorias, necesidad de conversión, estancia hospitalaria, complicaciones postoperatorias, necesidad de histerectomía, enfermedad residual, compromiso parametrial o de márgenes, conteo y estado de los ganglios, resultados obstétricos, recaídas, muerte y tiempo de seguimiento. La información se tomó de las historias clínicas las cuales fueron revisadas por dos de los investigadores y fue almacenada en una hoja de cálculo elaborada en Excel 2007. Se presentan medidas de tendencia central y dispersión para variables cuantitativas y frecuencias absolutas y proporciones para variables categóricas. Se utilizó el programa estadístico Stata $13.0 \AA$.

\section{RESULTADOS}

Trece pacientes fueron llevadas a traquelectomía radical laparoscópica, 12 pacientes $(92 \%)$ tenían tamaños tumorales iguales o menores a $2 \mathrm{~cm}$; una paciente con un tumor cervical de $3 \mathrm{~cm}$ recibió quimioterapia neoadyuvante con una disminución del $50 \%$ luego del tratamiento. El tipo histológico más frecuente fue el carcinoma de células escamosas en 7 pacientes (52\%) seguido de adenocarcinoma. El $100 \%$ de ellas tenía como parte del estudio preoperatorio una resonancia magnética nuclear. La duración media del procedimiento fue de 240 minutos (130-340 min). Como complicaciones intraoperatorias, se presentaron 2 cistotomías, 
las cuales fueron corregidas intraoperatoriamente. No hubo necesidad de conversión a laparotomía ni de administrar transfusiones sanguíneas. El sangrado promedio fue de $100 \mathrm{ml}(50-200 \mathrm{ml})$ y la estancia hospitalaria fluctuó entre de 1 y 3 días. La preservación de las arterias uterinas se llevó a cabo solo en una paciente a criterio del médico tratante. La colocación de cerclaje durante el mismo procedimiento también se realizó en una sola paciente, sin embargo este fue retirado a los 8 meses del postoperatorio.

En cuanto a los resultados de patología, el recuento ganglionar medio fue de 16 ganglios (1024 ganglios), todos ellos negativos al igual que los parametrios. El 62\% (8 pacientes) presentaron enfermedad residual en la pieza definitiva. Aunque ninguna paciente requirió terapia adyuvante, en 2 pacientes no se logro preservar la fertilidad y necesitaron ser llevadas a histerectomía laparoscópica; una de ellas por un margen positivo en el estudio en parafina (reportado como negativo en la biopsia por congelación) y la otra porque presentó una necrosis cornual como complicación postoperatoria a los 5 días de la traquelectomía.

En lo relacionado con las complicaciones postoperatorias se presentó una fístula vesicovaginal en una de las pacientes que tuvo cistotomía, la cual se manejó de manera expectante con sonda vesical con resolución a las 16 semanas del procedimiento quirúrgico. Dos pacientes (16\%) presentaron linfoquistes pélvicos que no requirieron ninguna intervención. Ninguna de la pacientes reportó disfunción vesical posterior a la cirugía ni se presentaron casos de estenosis del neo - cérvix (Tabla I).

\section{Tabla I
CARACTERÍSTICAS DEMOGRÁFICASY QUIRÚRGICAS DE LAS PACIENTES}

\begin{tabular}{|c|c|c|}
\hline Variable & n (\%) & Media (rango) \\
\hline Edad (años) & & $30(25-42)$ \\
\hline IMC (peso/talla $\left.{ }^{2}\right)$ & & $22,52(19,47-27,73)$ \\
\hline $\begin{array}{l}\text { Paridad: } \\
\text { - Cero } \\
\text { - Uno }\end{array}$ & $\begin{array}{l}10(77) \\
3(23)\end{array}$ & \\
\hline $\begin{array}{l}\text { Estadio FIGO IB1 } \\
\text { Tamaño: } \\
-<2 \mathrm{~cm} \\
->2 \mathrm{~cm}\end{array}$ & $\begin{array}{c}13(100) \\
12(92) \\
1(8)\end{array}$ & \\
\hline $\begin{array}{l}\text { Histología: } \\
\text { - Escamocelular } \\
\text { - Adenocarcinoma }\end{array}$ & $\begin{array}{l}7(54) \\
6(46)\end{array}$ & \\
\hline Tiempo quirúrgico (minutos) & & $240(130-340)$ \\
\hline Sangrado $(\mathrm{mL})$ & & $100(50-200)$ \\
\hline Transfusión & $0(0)$ & \\
\hline Preservación arterias uterinas & $1(8)$ & \\
\hline Colocación de cerclaje & $1(8)$ & \\
\hline Estancia hospitalaria (días) & & $1(1-3)$ \\
\hline $\begin{array}{l}\text { Complicaciones intraoperatoria } \\
\text { - Cistotomía }\end{array}$ & $2(16)$ & 15 \\
\hline $\begin{array}{l}\text { Complicaciones postoperatoria } \\
\text { - Linfoquistes } \\
\text { - Fístula vésico-vaginal }\end{array}$ & $\begin{array}{l}2(16) \\
1(8)\end{array}$ & \\
\hline Conteo ganglionar (n) & & $16(10-24)$ \\
\hline Ganglios positivos & $0(0)$ & \\
\hline Parametrios positivos & $0(0)$ & \\
\hline Enfermedad residual & $8(62)$ & \\
\hline Margen positivo & $1(8)$ & \\
\hline Adyuvancia & $0(0)$ & \\
\hline Preservación fertilidad & $11(85)$ & \\
\hline
\end{tabular}


Luego de un periodo de seguimiento promedio de 27,9 meses (8,3-64,4 meses), todas las pacientes han restaurado su ciclos menstruales y aunque no se han reportado embarazos, todas se encuentran libres de enfermedad y vivas al momento del último contacto.

\section{DISCUSIÓN}

A la fecha han sido reportados 247 casos de traquelectomía radical laparoscópica en la literatura. En Asia, Lee en 2003 (5), publica el primer reporte con 2 pacientes, posteriormente Cibula en 2005 (9), reporta el primer caso en Europa, y desde entonces múltiples autores han publicado sus resultados (10-19). El más grande de ellos por Park y cols con 79 pacientes (20), de las cuales 29 tenían tumores mayores a $2 \mathrm{cms}$ (Tablas II, III, IV).

Nuestra serie tiene resultados similares a los reportados por la mayoría de autores, a excepción de las series orientales en donde los tiempos hospitalarios son bastante prolongados, debido a razones sociales, culturales y de los sistemas de salud de esos países; sin embargo los tiempos quirúrgicos, el sangrado, los recuentos ganglionares y las complicaciones intra y posquirúrgicas son bastante similares (Tabla III).

Al comparar los resultados obstétricos de la técnica laparoscópica frente a la vaginal y abdominal encontramos que la tasa global de embarazos es de $23 \%, 24 \%$ y $16 \%$ respectivamente, mostrando bastante similitud con el abordaje vaginal. A la fecha, entre las pacientes a quienes se ha realizado la traquelectomía radical laparoscópica, se han producido 55 embarazos y 33 partos, lo que nos habla de la utilidad del procedimiento como cirugía preservadora de la fertilidad afirmando que no es menos efectiva que su contraparte vaginal y que ambas parecen ser superiores al abordaje por laparotomía (4).

Respecto a la seguridad oncológica, encontramos una tasa de recaída del $6 \%, 4,2 \%$ y $3,8 \%$, para el abordaje laparoscópico, vaginal y abdominal respectivamente. La mayor tasa de recurrencia observada en la vía laparoscópica puede verse afectada por el estudio de Park (20) de 2014 que incluye un número significativo de pacientes con tamaños tumorales mayores a $2 \mathrm{~cm}$.

La tasa de muerte por cáncer es de 1,7\%, 2,9\% y $0,4 \%$ para el procedimiento laparoscópico, vaginal y abdominal, respectivamente, lo que demuestra que no se pone en riesgo a la paciente desde el punto de vista oncológico, mientras se mantiene la posibilidad de fertilidad en el futuro.

Este estudio tiene ciertas limitaciones como son el escaso número de pacientes, su naturaleza retrospectiva, el corto tiempo de seguimiento, el hecho de que solo una paciente esté buscando quedar embarazada y que se trata de la experiencia de un solo centro.

\section{CARACTERÍSTICAS DEMOGRÁFICAS SEGÚN LA LITERATURA}

\begin{tabular}{lccccccccc}
\hline Autor & $\mathrm{n}$ & $\begin{array}{c}\text { Edad (años) } \\
\text { Media (rango) }\end{array}$ & $\mathrm{SC}$ & $\begin{array}{c}\text { Histología } \\
\text { AC }\end{array}$ & IA2 & IB1 & $\begin{array}{c}\text { Estadio } \\
\text { IB2 }\end{array}$ & IIA & $\begin{array}{c}\text { ILV + } \\
\mathrm{n}\end{array}$ \\
\hline Lee & 2 & $32(30-24)$ & 2 & 0 & 0 & 2 & 0 & 0 & $\mathrm{ND}$ \\
Cibula & 1 & $36(\mathrm{ND})$ & 1 & 0 & 0 & 1 & 0 & 0 & $\mathrm{ND}$ \\
Bafghi & 6 & $30(21-38)$ & 5 & 0 & 2 & 4 & 0 & 0 & 6 \\
Cibula & 3 & $\mathrm{ND}$ & $\mathrm{ND}$ & $\mathrm{ND}$ & $\mathrm{ND}$ & $\mathrm{ND}$ & $\mathrm{ND}$ & $\mathrm{ND}$ & $\mathrm{ND}$ \\
Park & 4 & $29(25-33)$ & 4 & 0 & 1 & 3 & 0 & 0 & 0 \\
Martin & 9 & $32(\mathrm{ND})$ & 6 & 3 & 2 & 7 & 0 & 0 & $\mathrm{ND}$ \\
Kim & 27 & $29(22-37)$ & 20 & 6 & 0 & 26 & 0 & 1 & 0 \\
Liu & 6 & $\mathrm{ND}(21-32)$ & 4 & 2 & 4 & 2 & 0 & 0 & 0 \\
Wang & 1 & $30(\mathrm{ND})$ & 1 & 0 & 1 & 0 & 0 & 0 & 0 \\
Lu & 25 & $29(22-34)$ & 25 & 0 & 10 & 15 & 0 & 0 & 2 \\
Ebisawa & 56 & $32(22-42)$ & 42 & 12 & 4 & 52 & 0 & 0 & 14 \\
Kucukmetin & 11 & $28(25-40)$ & 5 & 6 & 0 & 11 & 0 & 0 & 3 \\
Park & 79 & $31(20-40)$ & 60 & 18 & 4 & 72 & 2 & 1 & 12 \\
Saadi & 4 & $26(\mathrm{ND})$ & 2 & 1 & 0 & 4 & 0 & 0 & ND \\
Actual & 13 & $30(\mathrm{ND})$ & 7 & 6 & 0 & 12 & 0 & 0 & 0 \\
\hline
\end{tabular}

ILV: Invasión linfo-vascular. ND: No disponible. SC: Escamocelular. AC: Adenocarcinoma 


\section{CARACTERÍSTICAS QUIRÚRGICAS SEGÚN LA LITERATURA}

\begin{tabular}{lccccc}
\hline Autor & $\begin{array}{c}\text { Tiempo quirúrgico }(\mathrm{min}) \\
\text { Media (rango) }\end{array}$ & $\begin{array}{c}\text { Sangrado }(\mathrm{mL}) \\
\text { Media (rango) }\end{array}$ & $\begin{array}{c}\text { Transfusión } \\
\mathrm{n}\end{array}$ & $\begin{array}{c}\text { Ganglios } \\
\text { Media (rango) }\end{array}$ & $\begin{array}{c}\text { Estancia (días) } \\
\text { Media (rango) }\end{array}$ \\
\hline Lee & $414(398-430)$ & $650(400-900)$ & 0 & $35(20-50)$ & $12,5(7-18)$ \\
Cibula & $250(\mathrm{ND})$ & $250(\mathrm{ND})$ & 0 & $26(\mathrm{ND})$ & $6(\mathrm{ND})$ \\
Bafghi & $206(180-247)$ & $99(\mathrm{ND})$ & $\mathrm{ND}$ & $18(11-34)$ & $4,5(4-5)$ \\
Cibula & 210 & $400(\mathrm{ND})$ & $\mathrm{ND}$ & $24(\mathrm{ND})$ & $3(\mathrm{ND})$ \\
Park & $250(238-263)$ & $185(60-280)$ & 0 & $\mathrm{ND}$ & $8(\mathrm{ND})$ \\
Martin & $270(\mathrm{ND})$ & $\mathrm{ND}$ & 0 & $18(\mathrm{ND})$ & $5,2(\mathrm{ND})$ \\
Kim & $290(120-520)$ & $332(50-1000)$ & 6 & $25,7(8-50)$ & $9(4-18)$ \\
Liu & $185(155-210)$ & $105(60-120)$ & $\mathrm{ND}$ & $\mathrm{ND}$ & $18(15-23)$ \\
Wang & $280(\mathrm{ND})$ & $200(\mathrm{ND})$ & 0 & $12(\mathrm{ND})$ & $8(\mathrm{ND})$ \\
Lu & $232(180-340)$ & $120(50-200)$ & 0 & $24(13-37)$ & $3(2-4)$ \\
Ebisawa & $349(215-640)$ & $300(75-1540)$ & 0 & $32(14-55)$ & $17(7-47)$ \\
Kucukmetin & $320(210-410)$ & $85(50-3000)$ & 1 & $19(10-30)$ & $4(4-6)$ \\
Park & $291(120-710)$ & $393(50-1500)$ & 17 & $\mathrm{ND}$ & $9(3-28)$ \\
Saadi & $255(210-240)$ & $\mathrm{ND}$ & 0 & $18(15-20)$ & $1,3(1-1,5)$ \\
Actual & $241(130-340)$ & $95(50-200)$ & 0 & $16(10-24)$ & $1(1-3)$ \\
\hline
\end{tabular}

ND: No disponible

Tabla IV

CARACTERÍSTICAS OBSTÉTRICAS Y ONCOLÓGICAS SEGÚN LA LITERATURA

\begin{tabular}{lccccccc}
\hline Autor & $\begin{array}{c}\text { Seguimiento (meses) } \\
\text { Media (rango) }\end{array}$ & $\begin{array}{c}\text { Adyuvancia } \\
\mathrm{n}\end{array}$ & $\begin{array}{c}\text { Embarazo } \\
\mathrm{n}\end{array}$ & $\begin{array}{c}\text { Aborto } \\
\mathrm{n}\end{array}$ & $\begin{array}{c}\text { Parto } \\
\mathrm{n}\end{array}$ & $\begin{array}{c}\text { Recaída } \\
\mathrm{n}\end{array}$ & $\begin{array}{c}\text { Muerte } \\
\mathrm{n}\end{array}$ \\
\hline Lee & $10,5(9-12)$ & 0 & $\mathrm{ND}$ & $\mathrm{ND}$ & $\mathrm{ND}$ & 0 & 0 \\
Cibula & $4(\mathrm{ND})$ & 0 & 0 & 0 & 0 & 0 & 0 \\
Bafghi & $38(19-55)$ & 1 & 2 & 1 & 1 & 1 & 1 \\
Cibula & $21(\mathrm{ND})$ & $\mathrm{ND}$ & $\mathrm{ND}$ & $\mathrm{ND}$ & $\mathrm{ND}$ & $\mathrm{ND}$ & $\mathrm{ND}$ \\
Park & $34(27-37)$ & 0 & 0 & 0 & 0 & 1 & 0 \\
Martin & $28(6-32)$ & 0 & 2 & 0 & 1 & 1 & 0 \\
Kim & $31(1-58)$ & 0 & 3 & 2 & 1 & 1 & 1 \\
Liu & $\mathrm{ND}(8-20)$ & $\mathrm{ND}$ & 1 & $\mathrm{ND}$ & $\mathrm{ND}$ & 0 & 0 \\
Wang & $14(\mathrm{ND})$ & 0 & 0 & 0 & 0 & 0 & 0 \\
Lu & $66(1-82)$ & 0 & 9 & 3 & 4 & 13 & 1 \\
Ebisawa & $60(4-138)$ & 3 & 21 & 7 & $\mathrm{ND}$ & 0 & 0 \\
Kucukmetin & $9(1-20)$ & 0 & 0 & $\mathrm{ND}$ & 13 & 0 \\
Park & $44(3-105)$ & 9 & 17 & 4 & $\mathrm{ND}$ & 0 & 0 \\
Saadi & $9(5-20)$ & 0 & $\mathrm{ND}$ & $\mathrm{ND}$ & 0 & 0 \\
Actual & $14,6(1-42)$ & 0 & 0 & 0 & 0 & 0 \\
\hline
\end{tabular}

ND: No disponible 


\section{CONCLUSIONES}

La traquelectomía radical laparoscópica es una técnica segura desde el punto de vista oncológico, que brinda la posibilidad de preservar la fertilidad a pacientes con cáncer temprano de cuello uterino con resultados bastante similares a sus contrapartes vaginal y abdominal.

Esperamos con esta publicación, motivar a los ginecólogos oncólogos del continente, que con más frecuencia están realizando procedimientos mínimamente invasivos, a incorporar esta técnica dentro de las alternativas para ofrecer a sus pacientes.

DECLARACIÓN: Parte de este trabajo fué presentado como "free presentation" en el 42 Global Congress on Minimally Invasive Gynecology, en Washington, del 10 al 14 de noviembre de 2013.

\section{REFERENCIAS}

1. Dargent D BJ, Roy M, Remi I. Pregnancies following radical trachelectomy for invasive cervical cancer. Gynecol Oncol 1994;52:105.

2. Ribeiro Cubal AF, Ferreira Carvalho JI, Costa MF, Branco AP. Fertility-sparing surgery for early-stage cervical cancer. Int J Surg Oncol 2012;2012:936534.

3. Smith JR, Boyle DC, Corless DJ, Ungar L, Lawson $A D$, Del Priore $G$, et al. Abdominal radical trachelectomy: a new surgical technique for the conservative management of cervical carcinoma. Br J Obstet Gynaecol 1997; 104:1196-200.

4. Pareja R, Rendon GJ, Sanz-Lomana CM, Monzon $\mathrm{O}$, Ramirez PT. Surgical, oncological, and obstetrical outcomes after abdominal radical trachelectomy - a systematic literature review. Gynecol Oncol 2013;131:77-82.

5. Lee CL, Huang KG, Wang CJ, Yen CF, Lai CH. Laparoscopic radical trachelectomy for stage lb1 cervical cancer. J Am Assoc Gynecol Laparosc 2003;10:1115.

6. Persson J, Imboden S, Reynisson P, Andersson B, Borgfeldt C, Bossmar T. Reproducibility and accuracy of robot-assisted laparoscopic fertility sparing radical trachelectomy. Gynecol Oncol 2012;127:484-8.

7. NCCN. The National Comprehensive Cancer Network Clínical Practice Guidelines in Oncology. Cervical Cancer. CERV-2-3. Washington: Version 1. 2015.
8. Rendon GJ, Ramirez PT, Frumovitz M, Schmeler KM, Pareja R. Laparoscopic radical trachelectomy. JSLS 2012;16:503-7.

9. Cibula D, Ungar L, Palfalvi L, Bino B, Kuzel D. Laparoscopic abdominal radical trachelectomy. Gynecol Oncol 2005;97:707-9.

10. Cibula D, Slama J, Svarovsky J, Fischerova D, Freitag $\mathrm{P}$, Zikan $\mathrm{M}$, et al. Abdominal radical trachelectomy in fertility-sparing treatment of early-stage cervical cancer. Int J Gynecol Cancer 2009;19:1407-11.

11. Bafghi A, Castaigne D, Pomel C. [Radical trachelectomy: From the laparoscopic approach to the vaginal route]. J Gynecol Obstet Biol Reprod (Paris) 2006;35:696-701.

12. Park NY, Chong GO, Cho YL, Park IS, Lee YS. Total laparoscopic nerve-sparing radical trachelectomy. J Laparoendosc Adv Surg Tech A 2009;19:53-8.

13. Kim JH, Park JY, Kim DY, Kim YM, Kim YT, Nam JH. Fertility-sparing laparoscopic radical trachelectomy for young women with early stage cervical cancer. BJOG 2010;117:340-7.

14. Martin A, Torrent A. Laparoscopic nerve-sparing radical trachelectomy: surgical technique and outcome. J Minim Invasive Gynecol 2010;17:37-41.

15. Wang YF, Chen GW, Li WS, Weng HN, Lu XG. Total laparoscopic radical trachelectomy with ascending branches of uterine arteries preservation. Chin Med J (Engl) 2011;124:469-71.

16. Lu Q, Zhang Y, Liu C, Wang S, Guo S, Zhang Z. Total laparoscopic radical trachelectomy in the treatment of early squamous cell cervical cancer: a retrospective study with 8-year follow-up. Gynecol Oncol 2013;130:275-9.

17. Ebisawa K, Takano M, Fukuda M, Fujiwara K, Hada $\mathrm{T}$, Ota $\mathrm{Y}$, et al. Obstetric outcomes of patients undergoing total laparoscopic radical trachelectomy for early stage cervical cancer. Gynecol Oncol 2013;131:83-6.

18. Kucukmetin A, Biliatis I, Ratnavelu N, Patel A, Cameron I, Ralte A, et al. Laparoscopic radical trachelectomy is an alternative to laparotomy with improved perioperative outcomes in patients with early-stage cervical cancer. Int J Gynecol Cancer 2014;24:13540.

19. Saadi JM, Perrotta M, Orti R, Salvo G, Giavedoni ME, Gogorza S, Testa R, et al. Laparoscopic Radical Trachelectomy: Technique, Feasibility, and Outcomes. JSLS 2015; 19:e2013

20. Park JY, Joo WD, Chang SJ, Kim DY, Kim JH, Kim $\mathrm{YM}$, et al. Long-term outcomes after fertility-sparing laparoscopic radical trachelectomy in young women with early-stage cervical cancer: an Asan Gynecologic Cancer Group (AGCG) study. J Surg Oncol 2014;110:252-7. 